\title{
Real-Time Audio-to-Score Alignment Using Particle Filter for Coplayer Music Robots
}

\author{
Takuma Otsuka, ${ }^{1}$ Kazuhiro Nakadai, ${ }^{2,3}$ Toru Takahashi, ${ }^{1}$ Tetsuya Ogata, ${ }^{1}$ \\ and Hiroshi G. Okuno ${ }^{1}$ \\ ${ }^{1}$ Graduate School of Informatics, Kyoto University, Kyoto 606-8501, Japan \\ ${ }^{2}$ Honda Research Institute Japan, Co., Ltd., Wako, Saitama 351-0114, Japan \\ ${ }^{3}$ Graduate School of Information Science and Engineering, Tokyo Institute of Technology, Tokyo 152-8550, Japan
}

Correspondence should be addressed to Takuma Otsuka, ohtsuka@kuis.kyoto-u.ac.jp

Received 16 September 2010; Accepted 2 November 2010

Academic Editor: Victor Lazzarini

Copyright (C) 2011 Takuma Otsuka et al. This is an open access article distributed under the Creative Commons Attribution License, which permits unrestricted use, distribution, and reproduction in any medium, provided the original work is properly cited.

\begin{abstract}
Our goal is to develop a coplayer music robot capable of presenting a musical expression together with humans. Although many instrument-performing robots exist, they may have difficulty playing with human performers due to the lack of the synchronization function. The robot has to follow differences in humans' performance such as temporal fluctuations to play with human performers. We classify synchronization and musical expression into two levels: (1) melody level and (2) rhythm level to cope with erroneous synchronizations. The idea is as follows: When the synchronization with the melody is reliable, respond to the pitch the robot hears, when the synchronization is uncertain, try to follow the rhythm of the music. Our method estimates the score position for the melody level and the tempo for the rhythm level. The reliability of the score position estimation is extracted from the probability distribution of the score position. The experimental results demonstrate that our method outperforms the existing score following system in 16 songs out of 20 polyphonic songs. The error in the prediction of the score position is reduced by $69 \%$ on average. The results also revealed that the switching mechanism alleviates the error in the estimation of the score position.
\end{abstract}

\section{Introduction}

Music robots capable of, for example, dancing, singing, or playing an instrument with humans will play an important role in the symbiosis between robots and humans. Even people who do not speak a common language can share a friendly and joyful time through music not withstanding age, region, and race that we belong to. Music robots can be classified into two categories; entertainment-oriented robots like the violinist robot [1] exhibited in the Japanese booth at Shanghai Expo or dancer robots, and coplayer robots for natural interaction. Although the former category has been studied extensively, our research aims at the latter category, that is, a robot capable of musical expressiveness in harmony with humans.

Music robots should be coplayers rather than entertainers to increase human-robot symbiosis and achieve a richer musical experience. Their music interaction requires two important functions: synchronization with the music and generation of musical expressions, such as dancing or playing a musical instrument. Many instrument-performing robots such as those presented in [1-3] are only capable of the latter function, as they may have difficulty playing together with human performers. The former function is essential to promote the existing unidirectional entertainment to bidirectional entertainment.

We classify synchronization and musical expression into two levels: (1) the rhythm level and (2) the melody level. The rhythm level is used when the robot loses track of what part of a song is being performed, and the melody level is used when the robot knows what part is being played. Figure 1 illustrates the two-level synchronization with music.

When humans listen to a song being unaware of the exact part, they try to follow the beats by imagining a corresponding metronome, and stomp their feet, clap their hands, or scat to the rhythm. Even if we do not know the song 


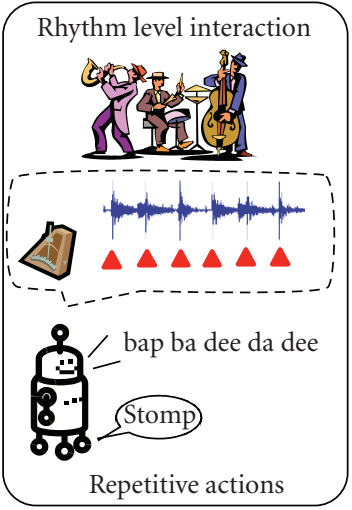

(a)

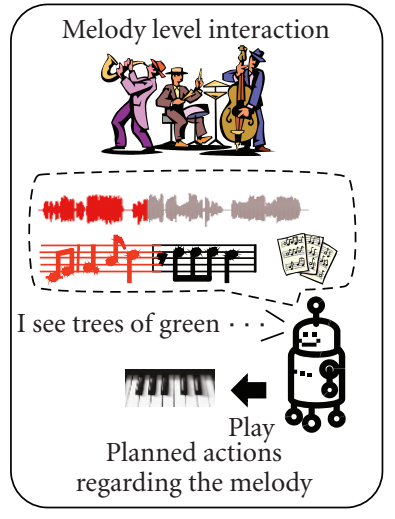

(b)
FIGURE 1: Two levels in musical interactions.

or the lyrics to sing, we can still hum the tune. On the other hand, when we know the song and understand which part is being played, we can also sing along or dance to a certain choreography. Two issues arise in achieving the two-layer synchronization and musical expression. First, the robot must be able to estimate the rhythm structure and the current part of the music at the same time. Second, the robot needs a confidence in how accurately the score position is estimated, hereafter referred to as an estimation confidence, to switch its behavior between the rhythm level and melody level.

Since most existing music robots that pay attention to the onset of a human's musical performance have focused on the rhythm level, their musical expressions are limited to repetitive or random expressions such as drumming [4], shaking their body [5], stepping, or scatting [6, 7]. Pan et al. developed a humanoid robot system that plays the vibraphone based on visual and audio cues [8]. This robot only pays attention to onset of human-played vibraphone. If the robot recognizes the pitch of human's performance, the ensemble will be enriched. A percussionist robot called Haile developed by Weinberg and Driscoll [9] uses MIDI signals to account for the melody level. However, this approach limits the naturalness of the interaction because live performances with acoustic instruments or singing voices cannot be de scribed by MIDI signals. If we stick to MIDI signals, we would have to develop a conversion system that can take any musical audio signal, including singing voices, and convert it to MIDI representation.

An incremental audio-to-score alignment [10] was previously introduced for the melody level for the purpose of a robot singer [11], but this method will not work if the robot fails to track the performance. The most important principle in designing a coplayer robot is robustness to the score fol lower's errors and to try to recover from them to make en semble performances more stable.

This paper presents a score following algorithm that conforms to the two-level model using a particle filter [12]. Our method estimates the score position for the melody level and tempo (speed of the music) for the rhythm level.
The estimation confidence is determined from the probability distribution of the score position and tempo. When the estimation of the score position is unreliable, only tempo is reported, in order to prevent the robot from performing incorrectly; when the estimation is reliable, the score position is reported.

\section{Requirements in Score Following for Musical Ensemble with Human Musicians}

Music robots have to not only follow the music but also predict upcoming musical notes for the following reasons. (1) A musical robot needs some temporal overhead to move its arms or actuators to play a musical instrument. To play in synchronization with accompanying human musicians, the robot has to start moving its arm in advance. This overhead also exists in MIDI synthesizers. For example, Murata et al. [7] reports that it takes around 200 ( $\mathrm{ms}$ ) to generate a singing voice using the singing voice synthesizer VOCALOID [13]. Ordinary MIDI synthesizers need 5-10 (ms) to synthesize instrumental sounds. (2) In addition, the score following process itself takes some time, at least 200-300 (ms) for our method. Therefore, the robot is only aware of the past score position. This also makes the prediction mandatory.

Another important requirement is the robustness against the temporal fluctuation in the human's performance. The coplayer robot is required to follow the human's performance even when the human accompanist varies his/her speed. Humans often changes his/her tempo in their performance for richer musical expressions.

2.1. State-of-the-Art Score Following Systems. Most popular score following methods are based on either dynamic time warping (DTW) $[14,15]$ or hidden Markov models (HMMs) $[16,17]$. Although the target of these systems is MIDI-based automatic accompaniment, the prediction of upcoming musical notes is not included in their score following model. The onset time of the next musical note is calculated by extrapolating those of the musical notes aligned with the score in the past.

Another score following method named Antescofo [18] uses a hybrid HMM and semi-Markov chain model to predict the duration of each musical note. However, this method reports the most likely score position whether it is reliable or not. Our idea is that using an estimation confidence of the score position to switch between behaviors would make the robot more intelligent in musical interaction.

Our method is similar to the graphical model-based method [19] in that it similarly models the transition of the score position and tempo. The difference is that this graphical model-based method follows the audio performance on the score by extracting the peak of the probability distribution over the score position and tempo. Our method approximates the probability distribution with a particle filter and extracts the peak as well as uses the shape of the distribution to derive an estimation confidence for two-level switching. 
A major difference between HMM-based methods and our method is how often a score follower updates the score position. HMM-based methods [16-18] update the estimated score position for each frame of short-time Fourier transform. Although this approach can naturally assume the transients of each musical note, for example, the onset, sustain, and release, the estimation can be affected by some frames that contain unexpected signals, such as the remainder of previous musical notes or percussive sounds without a harmonic structure. In contrast, our method uses frames with a certain length to update the score position and tempo of the music. Therefore, our method is capable of estimating the score position robustly against the unexpected signals. A similar approach is observed in [20] in that their method uses a window of recent performance to estimate the score position.

Our method is an extension of the particle filter-based score following [21] with switching between the rhythm and melody level. This paper presents an improvement in the accuracy of the score following by introducing a proposal distribution to make the most of information provided by the musical score.

\subsection{Problem Statement. The problem is specified as follows:}

Input: incremental audio signal and the corresponding musical score,

Output: predicted score position, or the tempo

Assumption: the tempo is provided by the musical score with a margin of error.

The issues are (1) simultaneous estimation of the score position and tempo and (2) the design of the estimation confidence. Generally, the tempo given by the score and the actual tempo in the human performance is different partly due to the preference or interpretation of the song, or partly due to the temporal fluctuation in the performance. Therefore, some margin of error should be assumed in the tempo information.

We assume that the musical score provides the approximate tempo and musical notes that consist of a pitch and a relative length, for example, a quarter note. The purpose of score following is to achieve a temporal alignment between the audio signal and the musical score. The onset and pitch of each musical note are important cues for the temporal audio-to-score alignment. The onset of each note is more important than the end of the notes because onsets are easier to recognize, whereas the end of a note is sometimes vague, for example, at the last part of a long tone. Our method models the tempo provided by the musical score and the alignment of the onsets in the audio and score as a proposal distribution in a framework of a particle filter. The pitch information is modeled as observation probabilities of the particle filter.

We model this simultaneous estimation as a state-space model and obtain the solution with a particle filter. The advantages of the use of a particle filter are as follows: (1) It enables an incremental and simultaneous estimation of the score position and tempo. (2) Real-time processing is possible because the algorithm is easily implemented with multithreaded computing. Further potential advantages are discussed in Section 5.1.

\section{Score Following Using Particle Filter}

3.1. Overview of Particle Filter. A particle filter is an algorithm for incremental latent variable estimation given observable variables [12]. In our problem, the observable variable is the audio signal and the latent variables are the score position and tempo, or beat interval in our actual model. The particle filter approximates the simultaneous distribution of the score position and beat interval by the density of particles with a set of state transition probabilities, proposal probabilities, and observation probabilities. With the incremental audio input, the particle filter updates the distribution and estimates the score position and tempo. The estimation confidence is determined from the probability distribution. Figure 3 outlines our method. The particle filter outputs three types of information: the predicted score position, tempo, and estimation confidence. According to the estimation confidence, the system reports either both the score position and tempo or only the tempo.

Our switching mechanism is achieved by estimating the beat interval independently of the score position. In our method, each particle has the beat interval and score position as a pair of hypotheses. First, the beat interval of each particle is stochastically drawn using the normalized crosscorrelation of the observed audio signal and the prior tempo from the score, without using the pitches and onsets written in the score. Then, the score position is drawn using the beat interval previously drawn and the pitches and onsets from the score. Thus, when the estimation confidence is low, we only rely on the beat interval for the rhythm level.

3.2. Preliminary Notations. Let $X_{f, t}$ be the amplitude of the input audio signal in the time frequency domain with frequency $f(\mathrm{~Hz})$ and time $t$ (sec.), and let $k$ (beat, the position of quarter notes) be the score position. In our implementation, $t$ and $f$ are discretized by a short-time Fourier transform with a sampling rate $44100(\mathrm{~Hz})$, a window length of 2048 (pt), and a hop size of 441 (pt). Therefore, $t$ and $f$ are discretized at a 0.01 -second and 21.5$\mathrm{Hz}$ interval. The score is also divided into frames for the discrete calculation such that the length of a quarter note equals 12 frames to account for the resolution of sixteenthnote and triplets. Musical notes $m_{k}=\left[m_{k}^{1} \cdots m_{k}^{r_{k}}\right]^{T}$ are placed at $k$, and $r_{k}$ is the number of musical notes. Each particle $p_{n}^{i}$ has score position, beat interval, and weight: $p_{n}^{i}=\left(k_{n}^{i}, b_{n}^{i}, w_{n}^{i}\right)$, and $N$ is the number of particles, that is, $1 \leq i \leq N$. The unit for $k_{n}^{i}$ is a beat, and the unit for $b_{n}^{i}$ is seconds per a beat. $n$ denotes the filtering step.

At the $n$th step the following procedure is carried out: (1) state transition using the proposal distribution, (2) observation and audio-score matching, and (3) estimation of the tempo and the score position, and resampling of the particles. Figure 2 illustrates these steps. The size of each particle represents its weight. After the resampling 


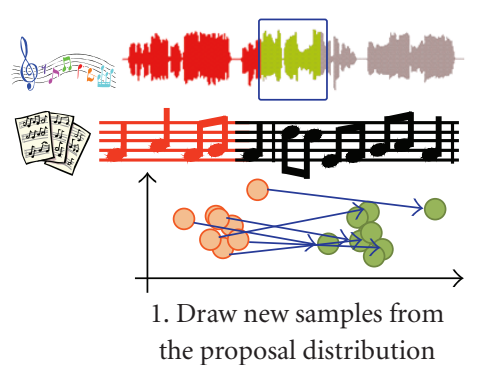

(a)

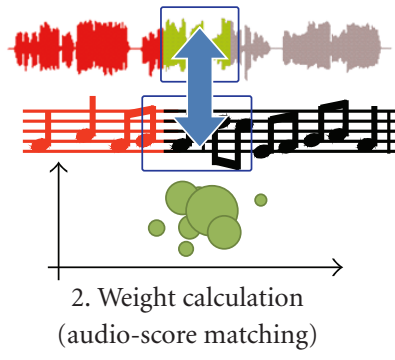

(b)

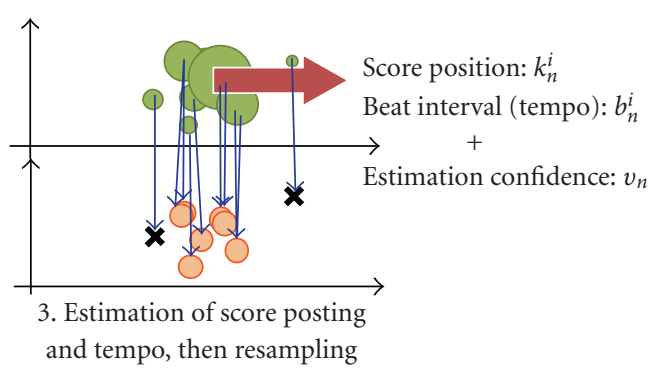

(c)

FIgURE 2: Overview of the score following using particle filter.

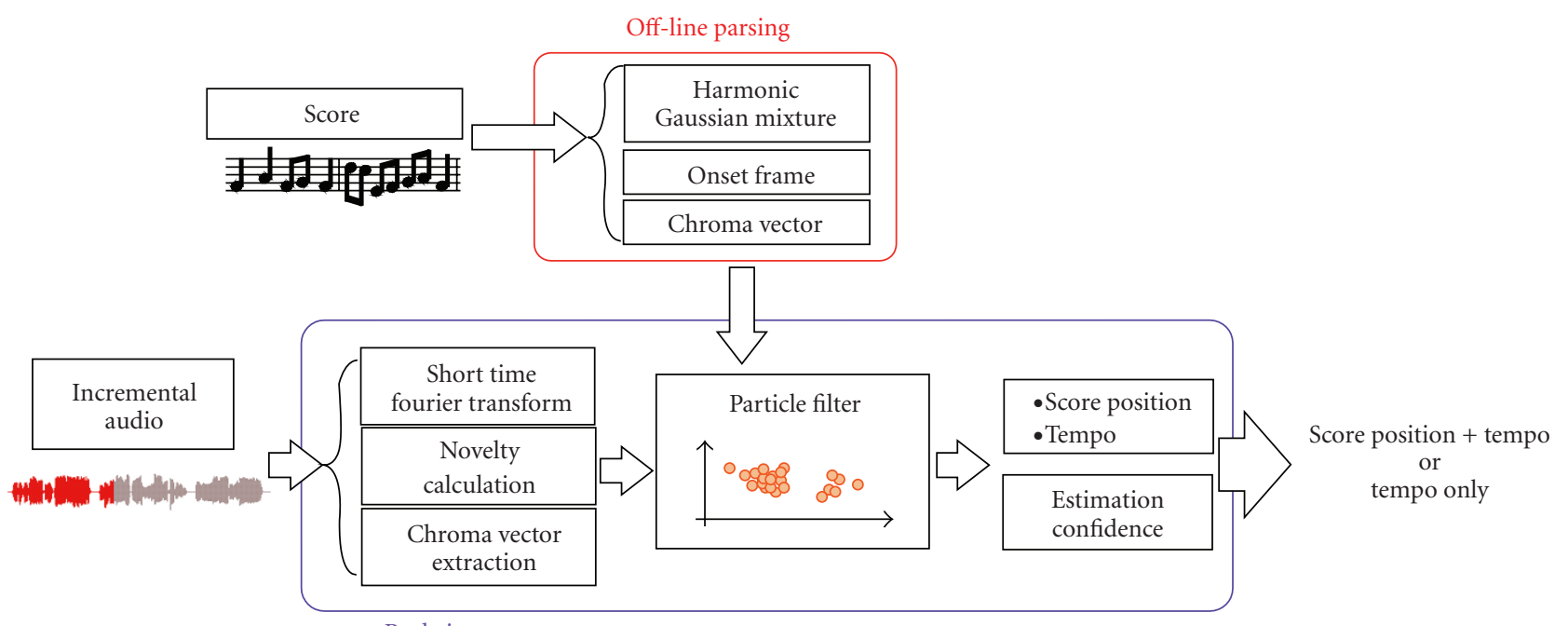

Real-time process

FIgURE 3: Two-level synchronization architecture.

step, the weights of all particles are set to be equal. Each procedure is described in the following subsections. These filtering procedures are carried out every $\Delta T$ (sec) and use an $L$-second audio buffer $X_{t}=\left[X_{f, \tau}\right]$ where $t-L<\tau \leq t$. In our configuration, $\Delta T=1(\mathrm{sec})$ and $L=2.5(\mathrm{sec})$. The particle filter estimates the score position $\hat{k}_{n}$ and the beat interval $\hat{b}_{n}$ at time $t=n \Delta T$.

3.3. State Transition Model. The updated score position and beat intervals of each particle are sampled from the following proposal distribution:

$$
\begin{gathered}
{\left[k_{n}^{i} b_{n}^{i}\right]^{T} \sim q\left(k, b \mid \mathbf{X}_{t}, \tilde{b}^{s}, o_{k}\right),} \\
q\left(k, b \mid \mathbf{X}_{t}, \tilde{b}^{s}, o_{k}\right)=q\left(b \mid \mathbf{X}_{t}, \tilde{b}^{s}\right) q\left(k \mid \mathbf{X}_{t}, o_{k}, b\right) .
\end{gathered}
$$

The beat interval $b_{n}^{i}$ is sampled from the proposal distribution $q\left(b \mid \mathbf{X}_{t}, \tilde{b}^{s}\right)$ that consists of the beat interval confidence based on normalized cross-correlation and the window function derived from the tempo $\widetilde{b}^{s}$ provided by the musical score. The score position $k_{n}^{i}$ is then sampled from the proposal distribution $q\left(k \mid \mathbf{X}_{t}, o_{k}, b_{n}^{i}\right)$ that uses the audio spectrogram $\mathbf{X}_{t}$, the onsets in the score $o_{k}$, and the sampled beat interval $b_{n}^{i}$.

3.3.1. Audio Preprocessing for the Estimation of the Beat Interval and Onsets. We make use of the Euclidean distance of Fourier coefficients in the complex domain [22] to calculate a likely beat interval from the observed audio signal $\mathbf{X}_{t}$ and onset positions in the audio signal. This method is chosen from many other onset detection methods as introduced in [23] because this method emphasizes onsets of many kinds of timbres, for example, wind instruments like flute or string instruments like guitar, with moderate computational cost. $\Xi_{f, t}$ in the following (2) is the distance between two adjacent Fourier coefficients in time frame. The more the distance is, the more the onset is likely to exist.

$$
\begin{gathered}
\Xi_{f, t}=\left\{X_{f, t}^{2}+X_{f, t-\Delta t}^{2}-2 X_{f, t} X_{f, t-\Delta t} \cos \left(\Delta \varphi_{f, t}\right)\right\}^{1 / 2}, \\
\Delta \varphi_{f, t}=\varphi_{f, t}-2 \varphi_{f, t-\Delta t}+\varphi_{f, t-2 \Delta t},
\end{gathered}
$$

where $\varphi_{f, t}$ is an unwrapped phase at the same frequency bin and time frame as $X_{f, t}$ in the complex domain. $\Delta t$ denotes the 
interval time of the short-time Fourier transform. When the signal is stable, $\Xi_{f, t} \approx 0$ because $X_{f, t} \approx X_{f, t-\Delta t}$ and $\Delta \varphi_{f, t} \approx 0$.

3.3.2. Proposal Distribution for the Beat Interval. The beat interval is drawn from the following proposal:

$$
\begin{gathered}
b_{n}^{i} \sim q\left(b \mid \mathbf{X}_{t}, \tilde{b}^{s}\right) \\
q\left(b \mid \mathbf{X}_{t}, \tilde{b}^{s}\right) \propto R\left(b, \bar{\Xi}_{t}\right) \times \psi\left(b \mid \tilde{b}^{s}\right) .
\end{gathered}
$$

We obtain $\bar{\Xi}_{t}=\left[\bar{\Xi}_{m, \tau}\right]$, where $1 \leq m \leq 64$ and $t-L<$ $\tau \leq t$, by reducing the dimension of the frequency bins into 64 dimensions by 64 equally placed mel-filter banks. A linear scale frequency $f^{\mathrm{Hz}}$ is converted into a mel-scale frequency $f^{\mathrm{mel}}$ as

$$
f^{\mathrm{mel}}=1127 \log \left(1+\frac{f^{\mathrm{Hz}}}{700}\right)
$$

64 triangular windows are constructed with an equal width on the mel scale as

$$
\begin{gathered}
W_{m}\left(f^{\mathrm{mel}}\right)= \begin{cases}\frac{f^{\mathrm{mel}}-f_{m-1}^{\mathrm{mel}}}{f_{m}^{\mathrm{mel}}-f_{m-1}^{\mathrm{mel}}}, & f_{m-1}^{\mathrm{mel}} \leq f^{\mathrm{mel}}<f_{m}^{\mathrm{mel}}, \\
\frac{f_{m+1}^{\mathrm{mel}}-f^{\mathrm{mel}}}{f_{m+1}^{\mathrm{mel}}-f_{m}^{\mathrm{mel}}}, & f_{m}^{\mathrm{mel}} \leq f^{\mathrm{mel}}<f_{m+1}^{\mathrm{mel}}, \\
0, & \text { otherwise }\end{cases} \\
\qquad f_{m}^{\mathrm{mel}}=\frac{m}{64} f_{\mathrm{Nyq}}^{\mathrm{mel}}
\end{gathered}
$$

where (8) indicates the edges of each triangular window and $f_{\mathrm{Nyq}}^{\text {mel }}$ denotes the mel-scale frequency of the Nyquist frequency. The window function $W_{m}\left(f^{\mathrm{mel}}\right)$ when $m=64$ has only the top part in (7) because $f_{64+1}^{\mathrm{mel}}$ is not defined. Finally, we obtain $\bar{\Xi}_{m, \tau}$ by applying the window functions $W_{m}\left(f^{\mathrm{mel}}\right)$ to $\Xi_{f, \tau}$ as follows:

$$
\bar{\Xi}_{m, \tau}=\int W_{m}\left(f^{\mathrm{mel}}\right) \Xi_{f, \tau} d f
$$

where $f^{\text {mel }}$ is a mel-frequency corresponding to the linear frequency $f$. $f$ is converted into $f^{\text {mel }}$ by (6).

With this dimension reduction, the normalized cross correlation is less affected by the difference between each sound's spectral envelope. Therefore, the interval of onsets by any instrument and with any musical note is robustly emphasized. The normalized cross correlation is defined as

$$
R\left(b, \bar{\Xi}_{t}\right)=\frac{\int_{t-L}^{t} \sum_{m=1}^{64} \bar{\Xi}_{m, \tau} \bar{\Xi}_{m, \tau-b} d \tau}{\sqrt{\int_{t-L}^{t} \sum_{m=1}^{64} \bar{\Xi}_{m, \tau}^{2} d \tau \int_{t-L}^{t} \sum_{m=1}^{64} \bar{\Xi}_{m, \tau-b}^{2} d \tau}} .
$$

The window function is centered at $\tilde{b}^{s}$ the tempo specified by the musical score.

$$
\psi\left(b \mid \tilde{b}^{s}\right)= \begin{cases}1 & \left|\frac{60}{b}-\frac{60}{\tilde{b}^{s}}\right|<\theta \\ 0 & \text { otherwise. }\end{cases}
$$

where $\theta$ is the width of the window in beats per minute (bpm). A beat interval $b$ (sec/beat) is converted into a tempo value $m(\mathrm{bpm}=$ beat $/ \mathrm{min})$ by the equation

$$
m=\frac{60}{b} \text {. }
$$

Equation (11) limits the beat interval value of particles so as not to miss the score position by a false tempo estimation.

3.3.3. Proposal Distribution for the Score Position. The score position is sampled as

$$
\begin{aligned}
& k_{n}^{i} \sim q\left(k \mid \mathbf{X}_{t}, o_{k}, b_{n}^{i}\right), \\
& q\left(k \mid \mathbf{X}_{t}, o_{k}, b_{n}^{i}\right) \\
& \propto \begin{cases}\int_{t-L}^{t} \xi_{\tau} o_{\tilde{k}(\tau)} d \tau & \left(o_{\tilde{k}(\tau)}=1, \exists \tau \wedge k \in K\right), \\
1 & \left(o_{\tilde{k}(\tau)}=0, \text { for } \forall \tau \wedge k \in K\right), \\
0, & k \notin K,\end{cases} \\
& \xi_{t}=\int \begin{array}{ll}
\Xi_{f, t} d f . &
\end{array}
\end{aligned}
$$

The score onset $o_{k}=1$ when the onset of any musical note exists at $k$, otherwise $o_{k}=0 . \tilde{k}(\tau)$ is an aligned score position at time $\tau$ using the particle's beat interval $b_{n}^{i}: \tilde{k}(\tau)=k-(t-$ $\tau) / b_{n}^{i}$, assuming the score position is $k$ at time $t$. Equation (15) assigns high weight on the score position where the drastic change in the audio denoted by $\xi_{t}$ and onsets in the score $o_{\tilde{k}(\tau)}$ are well aligned. In case no onsets are found in the neighborhood in the score, a new score position $k_{n}^{i}$ is selected at random from the search area $K . K$ is set such that the center is at $k_{n-1}^{i}+\Delta T / b_{n}^{i}$ and the width is $3 \sigma_{k}$, where $\sigma_{k}$ is empirically set to 1 .

3.3.4. State Transition Probability. State transition probabilities are defined as follows:

$$
\begin{aligned}
& p\left(b, k \mid b_{n-1}^{i}, k_{n-1}^{i}\right) \\
& =\mathcal{N}\left(b \mid b_{n-1}^{i}, \sigma_{b}^{2}\right) \times \mathcal{N}\left(k \mid k_{n-1}^{i}+\frac{\Delta T}{b_{n}^{i}}, \sigma_{k}^{2}\right),
\end{aligned}
$$

where the variance for the beat interval transition $\sigma_{b}^{2}$ is empirically set to 0.2 . These probabilities are used for the weight calculation in (17).

3.4. Observation Model and Weight Calculation. At time $t$, a spectrogram $\mathbf{X}_{t}=\left[X_{f, \tau}\right](t-L<\tau \leq t)$ is used for the weight calculation. The weight of each particle at the $n$th step $w_{i, n}, 1 \leq i \leq N$ is calculated as

$$
w_{i, n}=\frac{p\left(\mathbf{X}_{t} \mid b_{n}^{i}, k_{n}^{i}\right) p\left(b, k \mid b_{n-1}^{i} k_{n-1}^{i}\right)}{q\left(b \mid \mathbf{X}_{t}, \tilde{b}^{s}\right)},
$$


where $p\left(b, k \mid b_{n-1}^{i} k_{n-1}^{i}\right)$ is defined in (16) and $q\left(b \mid \mathbf{X}_{t}, \tilde{b}^{s}\right)$ is defined in (5). The observation probability $p\left(\mathbf{X}_{t} \mid b_{n}^{i}, k_{n}^{i}\right)$ consists of three parts as

$$
p\left(\mathbf{X}_{t} \mid b_{n}^{i}, k_{n}^{i}\right) \propto w_{i, n}^{\mathrm{ch}} \times w_{i, n}^{\mathrm{sp}} \times w_{i, n}^{t}
$$

The two weights, the chroma vector weight $w_{i, n}^{\text {ch }}$ and spectrogram weight $w_{i, n}^{\mathrm{sp}}$, are measures of pitch information. The weight $w_{i, n}^{t}$ is a measure of temporal information. We use both the chroma vector similarity and the spectrogram similarity to estimate the score position because they have a complementary relationship. A chroma vector has 12 elements corresponding to the pitch name, $C, C \#, \ldots, B$. This is a convenient feature for audio-to-score matching because the chroma vector is easily derived from both the audio signal and the musical score. However, the elements of a chroma vector become ambiguous when the pitch is low due to the frequency resolution limit. The harmonic structure observed in the spectrogram alleviates this problem because it makes the pitch distinct in the higher frequency region.

3.4.1. Alignment of the Buffered Audio Signal with the Score. To match the spectrogram $X_{f, \tau}$, where $t-L<\tau \leq t$, the audio sequence is aligned with the corresponding score for each particle, as shown in Figure 4. Each frame of the spectrogram at time $\tau$ is assigned to the score frame $\tilde{k}(\tau)^{i}$ using the estimated score position $k_{n}^{i}$ and the beat interval (tempo) $b_{n}^{i}$ as

$$
\tilde{k}(\tau)^{i}=k_{n}^{i}-\frac{t-\tau}{b_{n}^{i}} .
$$

3.4.2. Chroma Vector Matching. The sequence of chroma vectors $\mathbf{c}_{\tau}^{a}=\left[c_{\tau, j}^{a}\right]^{T}, 1 \leq j \leq 12$ is calculated from the spectrum $X_{f, \tau}$ using band-pass filters $B_{j, o}(f)$ for each element [24] as

$$
c_{\tau, j}^{a}=\sum_{o=\text { Octlow }}^{\text {Octhi }} \int X_{f, \tau} B_{j, o}(f) d f,
$$

where $B_{j, o}(f)$ is the band-pass filter that passes a signal with $\log$-scale frequency $f_{j, o}^{\text {cent }}$ of the chroma class $j$ and the octave $o$. That is,

$$
f_{j, o}^{\text {cent }}=1200 \times o+100 \times(j-1) .
$$

A linear-scale frequency $f^{\mathrm{Hz}}$ is converted into the log-scale frequency $f^{\text {cent }}$ as

$$
f^{\text {cent }}=1200 \log _{2} \frac{f^{\mathrm{Hz}}}{440 \times 2^{3 / 12-5}} .
$$

Each band-pass filter $B_{j, o}(f)$ is defined as

$$
B_{j, o}\left(f^{\mathrm{Hz}}\right)=\frac{1}{2}\left(1-\cos \frac{2 \pi\left(f^{\mathrm{cent}}-\left(f_{j, o}^{\mathrm{cent}}-100\right)\right)}{200}\right),
$$

where $f_{j, o}^{\text {cent }}-100 \leq f^{\text {cent }} \leq f_{j, o}^{\text {cent }}+100$. The range of octaves are set Oct low $=3$ and $\mathrm{Oct}_{\text {hi }}=6$. The value of each element in the score chroma vector $\mathbf{c}_{k_{\tau}^{i}}^{s}$ is 1 when the score has a corresponding note between the octaves Oct $\mathrm{s}_{\text {low }}$ and Oct $\mathrm{Ch}_{\mathrm{hi}}$, and 0 otherwise. The range of the chroma vector is between $C$ note in octave 3 and $B$ note in octave 6 . Their fundamental frequencies are $131(\mathrm{~Hz})$ and $1970(\mathrm{~Hz})$, respectively.

The chroma weight $w_{i, n}^{\text {ch }}$ is calculated as

$$
w_{\mathrm{i}, n}^{\mathrm{ch}}=\frac{1}{L} \int_{t-L}^{t} \mathbf{c}_{\tau}^{a} \cdot \mathbf{c}_{\widetilde{k}(\tau)^{i}}^{s} d \tau
$$

Both vectors $b f c_{\tau}^{a}$ and $c_{\tilde{k}(\tau)^{i}}^{s}$ are normalized before applying them to (24).

3.4.3. Harmonic Structure Matching. The spectrogram weight $w_{i, n}^{\mathrm{sp}}$ is derived from the Kullback-Leibler divergence with regard to the shape of spectrum between the audio and the score.

$$
\begin{gathered}
w_{i, n}^{\mathrm{sp}}=\frac{1}{L} \int_{t-L}^{t}\left(\frac{1}{2}+\frac{1}{2} \tanh \frac{D_{i, \tau}^{\mathrm{KL}}-\bar{D}^{\mathrm{KL}}}{\nu}\right), \\
D_{i, \tau}^{\mathrm{KL}}=\int_{0}^{f_{\max }} X_{f, \tau} \log \frac{X_{f, \tau}}{\hat{X}_{f, \tilde{k}(\tau)^{i}}},
\end{gathered}
$$

where $D_{i, \tau}^{\mathrm{KL}}$ in $(26)$ is the dissimilarity between the audio and score spectrograms. Before calculating (26), the spectrum is normalized such that $\int_{0}^{f_{\max }} X_{f, \tau} d f=\int_{0}^{f_{\max }} \hat{X}_{f, \tilde{k}(\tau)^{i}} d f=1$. The range of the frequency for calculating the Kullback-Leibler divergence is limited under $f_{\max }(\mathrm{Hz})$ because most of the energy in the audio signal is located in low frequency region. We set the parameter as $f_{\max }=6000(\mathrm{~Hz})$. The positive value $D_{i}^{\mathrm{KL}}$ is mapped to the weight $w_{i, n}^{\mathrm{sp}}$ by (25) where the range of $w_{i, n}^{\mathrm{sp}}$ is between 0 and 1 . Here, the hyperbolic function is used with the threshold distance $\bar{D}^{\mathrm{KL}}=4.2$ and the tilt $v=0.8$ which are set empirically.

3.4.4. Preprocessing of the Musical Score. For the calculation of $w_{i, n}^{\mathrm{sp}}$, the spectrum $\hat{X}_{f, k}$ is generated from the musical score in advance of particle filtering by the harmonic Gaussian mixture model (GMM), the first term in

$$
\hat{X}_{f, k}=C_{\text {harm }} \sum_{r=1}^{r_{k}} \sum_{g=1}^{G} h(g) N\left(f ; g F\left(m_{k}^{r}\right), \sigma^{2}\right)+C_{\text {floor }} .
$$

In (27), $g$ is the harmonic index, $G$ is the number of harmonics, and $h(g)$ is the height of each harmonic. $F\left(m_{k}^{r}\right)$ is the fundamental frequency of note $m_{k}^{r}$ and the variance $\sigma^{2}$. Let $m$ be a note number used in the standard MIDI (Musical Instrument Digital Interface), $F(m)$ is derived as $F(m)=440 \times 2^{(m-69) / 12}$. The parameters are empirically set as $G=10, h(g)=0.2^{g}, \sigma^{2}=0.8$. To avoid zero divides in 


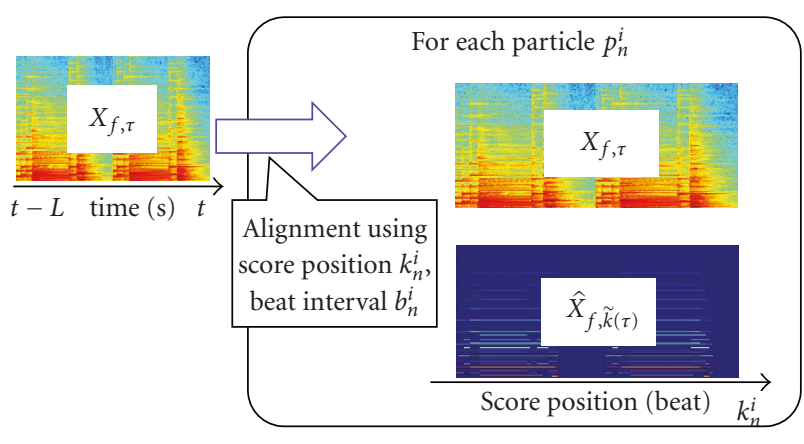

FIGURE 4: Weight calculation for pitch information.

(26), the constant factor $C_{\text {harm }}$ is set and the floor constant $C_{\text {floor }}$ is added to the score spectrogram such that

$$
\begin{gathered}
\int C_{\text {harm }} \sum_{r=1}^{r_{k}} \sum_{g=1}^{G} h(g) N\left(f ; g F\left(m_{k}^{r}\right), \sigma^{2}\right) d f=0.9, \\
C_{\text {floor }}=0.1 .
\end{gathered}
$$

3.4.5. Beat Interval Matching. The weight $w_{i, n}^{t}$ is the measure of the beat interval and obtained from the normalized cross correlation of the spectrogram through a shift by $b_{n}^{i}$ :

$$
w_{i, n}^{t}=R\left(b_{n}^{i}, \bar{\Xi}_{t}\right) \text {, }
$$

where $R\left(b_{n}^{i}, \bar{\Xi}_{t}\right)$ is defined in (10).

3.5. Estimation of Score Position and Beat Interval. After calculating the weight of all particles the score position $\hat{k}_{n}$ and the beat interval, equivalent to the tempo, $\hat{b}_{n}$ are estimated by averaging the values of particles that have more weight. We use the top $20 \%$ high-weight particles for this estimation.

$$
\begin{gathered}
\hat{k}_{n}=\sum_{i \in P_{20 \%}} \frac{w_{n}^{i} k_{n}^{i}}{W}, \\
\hat{b}_{n}=\sum_{i \in P_{20 \%}} \frac{w_{n}^{i} b_{n}^{i}}{W}, \\
W=\sum_{i \in P_{20 \%}} w_{n}^{i},
\end{gathered}
$$

where $P_{20 \%}$ is the set of indicis of the top 20\% high-weight particles. For example, when the number of particle $N=$ 1000 , the size of $P_{20 \%}$ is 200 .

Given the current score position $\hat{k}_{n}$ and beat interval $\hat{b}_{n}$, the score position $\Delta T$ ahead in time $\hat{k}_{n}^{\text {pred }}$ is predicted by the following equation:

$$
\hat{k}_{n}^{\text {pred }}=\hat{k}_{n}+\frac{\Delta T}{\hat{b}_{n}} \text {. }
$$

3.6. Resampling. After calculating the score position and beat interval with (31) and (32), the particles are resampled. In this procedure, particles with a large weight are likely to be selected many times, whereas those with a small weight are discarded because their score position is unreliable. A particle $p$ is drawn independently $N$ times from the distribution:

$$
P\left(p=p_{n}^{i}\right)=\frac{w_{n}^{i}}{\sum_{i=1}^{N} w_{n}^{i}} .
$$

After resampled, the weights of all particles are set to be equal.

3.7. Initial Probability Distribution. The initial particles at $n=0$ are set as follows: (1) draw $N$ samples of the beat interval $b_{0}^{i}$ value from a uniform distribution ranging from $\tilde{b}^{s}-60 / \theta$ to $\tilde{b}^{s}+60 / \theta$ where $\theta$ is the window width in (11). (2) Set the score position of each particle $k_{n}^{i}$ to 0.

3.8. Estimation Confidence of Score Following. The weight of local peaks of the probability distribution of the score position and the beat interval is used as the estimation confidence. Let $P_{2} \%$ be the set of indicis of the top $2 \%$ highweight particles in number, for example, $\left|P_{2 \%}\right|=20$ when $N=1000$. Particles $P_{2} \%$ are regarded as the local peak of the probability distribution. The estimation confidence $v_{n}$ is defined as

$$
v_{n}=\frac{\sum_{i \in P_{2 \%}} w_{n}^{i}}{\sum_{1 \leq i \leq N} w_{n}^{i}} .
$$

When $v_{n}$ is high, it means that high-weight particles are tracking a reliable hypothesis; when $v_{n}$ is low, particles fail to find out a remarkable hypethosis.

Based on this idea, switching the melody level and rhythm level is carried out as follows.

(1) First, the system is on the melody level, therefore it reports both the score position and tempo.

(2) If $v_{n}$ decreases such that (37) is satisfied, the system switches to the rhythm level and stops reporting the score position.

(3) If $v_{n}$ increases again and (37) is satisfied, the system switches back to the melody level and resumes reporting the estimated score position

$$
\begin{gathered}
v_{n}-v_{n-1}<-\gamma_{\mathrm{dec}}, \\
v_{n}-v_{n-1}>\gamma_{\text {inc }} .
\end{gathered}
$$

The parameters are empirically set as: $\gamma_{\mathrm{dec}}=0.08$ and $\gamma_{\mathrm{inc}}=$ 0.07 , respectively.

\section{Experimental Evaluation}

This section presents the prediction error of the score following in various conditions: (1) comparisons with Antescofo [25], (2) the effect of two-level synchronization, (3) the effect of the number of particles $N$, and (4) the effect of the width of window function $\theta$ in (11). Then, the computational cost of our algorithm is discussed in Section 4.3. 
TABle 1: Parameter settings.

\begin{tabular}{|c|c|c|c|}
\hline \multicolumn{2}{|l|}{ Denotation } & \multicolumn{2}{|c|}{ Value } \\
\hline Filtering interval & $\Delta T$ & 1 & $(\mathrm{sec})$ \\
\hline Audio buffer length & $L$ & 2.5 & $(\mathrm{sec})$ \\
\hline Score position variance & $\sigma_{k}^{2}$ & 1 & $\left(\right.$ beat $\left.^{2}\right)$ \\
\hline Beat duration variance & $\sigma_{b}^{2}$ & 0.2 & $\left(\sec ^{2} /\right.$ beat $\left.^{2}\right)$ \\
\hline $\begin{array}{l}\text { Upper limit in harmonic } \\
\text { structure matching }\end{array}$ & $f_{\max }$ & 6000 & $(\mathrm{~Hz})$ \\
\hline $\begin{array}{l}\text { Lower octave for chroma } \\
\text { vector extraction }\end{array}$ & Oct $_{\text {low }}$ & 3 & $(\mathrm{~N} / \mathrm{A})$ \\
\hline $\begin{array}{l}\text { Higher octave for chroma } \\
\text { vector extraction }\end{array}$ & Oct $_{\text {hi }}$ & 6 & $(\mathrm{~N} / \mathrm{A})$ \\
\hline
\end{tabular}

TABLE 2: Songs used for the experiments.

\begin{tabular}{cccl}
\hline Song ID & File name & Tempo $(\mathrm{bpm})$ & Instrumentsmark $^{1}$ \\
\hline 1 & RM-J001 & 150 & Pf \\
2 & RM-J003 & 98 & Pf \\
3 & RM-J004 & 145 & Pf \\
4 & RM-J005 & 113 & Pf \\
5 & RM-J006 & 163 & Gt \\
6 & RM-J007 & 78 & Gt \\
7 & RM-J010 & 110 & Gt \\
8 & RM-J011 & 185 & Vib \& Pf \\
9 & RM-J013 & 88 & Vib \& Pf \\
10 & RM-J015 & 118 & Pf \& Bs \\
11 & RM-J016 & 198 & Pf, Bs \& Dr \\
12 & RM-J021 & 200 & Pf, Bs, Tp \& Dr \\
13 & RM-J023 & 84 & Pf, Bs, Sax \& Dr \\
14 & RM-J033 & 70 & Pf, Bs, Fl \& Dr \\
15 & RM-J037 & 214 & Pf, Bs, Vo \& Dr \\
16 & RM-J038 & 125 & Pf, Bs, Gt, Tp \& Dr etc. \\
17 & RM-J046 & 152 & Pf, Bs, Gt, Kb \& Dr etc. \\
18 & RM-J047 & 122 & Kb, Bs, Gt \& Dr \\
19 & RM-J048 & 113 & Pf, Bs, Gt, Kb \& Dr etc. \\
20 & RM-J050 & 157 & Kb, Bs, Sax \& Dr \\
\hline
\end{tabular}

${ }^{1}$ abbreviations: Pf: Piano, Gt: Guitar, Vib: Vibraphone, Bs: Bass, Dr: Drums, Tp: Trumpet, Sax: Saxophone, Fl: Flute, Vo: Vocal, Kb: Keyboard.

4.1. Experimental Setup. Our system was implemented in $\mathrm{C}++$ with Intel $\mathrm{C}++$ Compiler on Linux with an Intel Corei7 processor. We used 20 jazz songs from the RWC Music Database [26] listed in Table 2. These are recordings of the actual humans' performance. Note that the musical scores are manually transcribed note for note. However, only the pitch and length of musical notes are the input for our method. We use the jazz songs as experimental materials because a variety of musical instruments are included in the songs as shown in Table 2. The problem that the scores for jazz music do not always specify all musical notes is discussed in Section 5.1. The average length of these songs is around 3 minutes. The sampling rate was $44100(\mathrm{~Hz})$ and the Fourier transform was executed with a 2048 (pt) window length and 441 (pt) window shift. The parameter settings are listed in Table 1 .

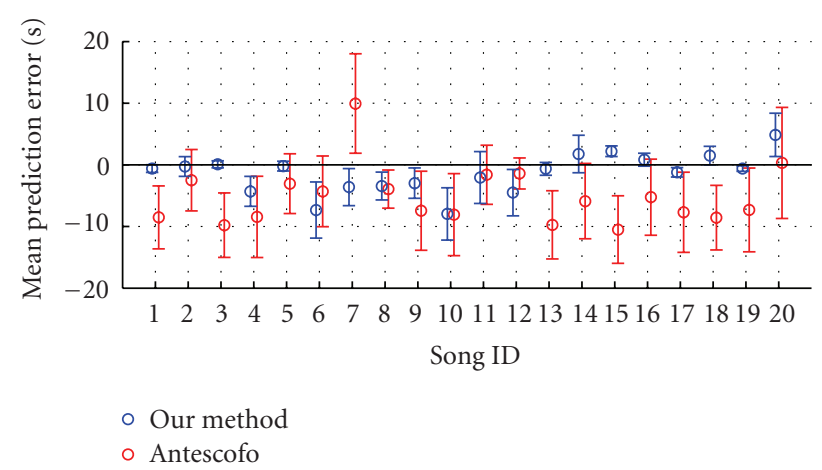

Figure 5: Mean prediction errors in our method and Antescofo: the number of particles $N$ is 1500 , the width of the tempo window $\theta$ is 15 (bpm).

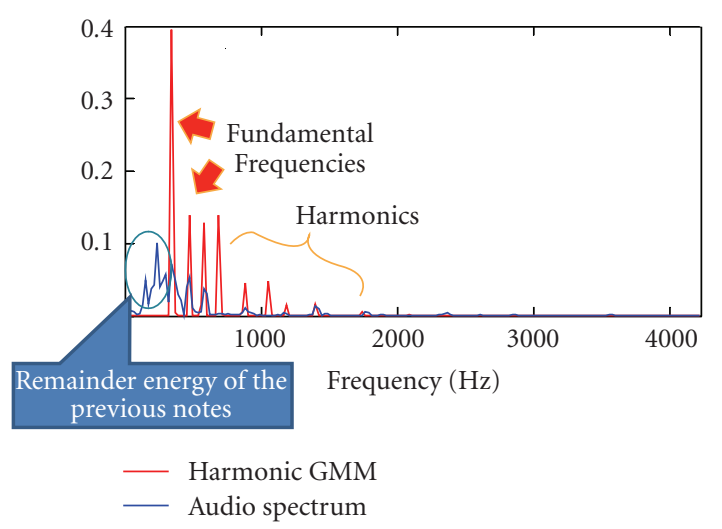

Figure 6: Comparison between harmonic GMM generated by the score and the actual audio spectrum.

4.2. Score Following Error. At $\Delta T$ intervals, our system predicts the score position $\Delta T$ (sec) ahead as $\hat{k}_{n}^{\text {pred }}$ in (33) when the current time is $t$. Let $s(k)$ be the ground truth time at beat $k$ in the music. $s(k)$ is defined for positive continuous $k$ by linear interpolation of musical event times. The prediction error $e^{\text {pred }}(t)$ is defined as

$$
e^{\text {pred }}(t)=t+\Delta T-s\left(\hat{k}_{n}^{\text {pred }}\right) .
$$

Positive $e^{\text {pred }}(t)$ means the estimated score position is behind of the true position by $e^{\text {pred }}(t)(\mathrm{sec})$.

\subsubsection{Our Method versus Hybrid HMM-Based Score Following} Method. Figure 5 shows the errors in the predicted score positions for 20 songs when the number of particles $N$ is 1500 and the width of the tempo window $\theta$ corresponds to 15 (bpm). The comparison between our method in blue plots and Antescofo [25] in red plots. The mean values of our method is calculated by averaging all prediction errors both on the rhythm level and on the melody level. This is because Figure 5 is intended to compare the particle filterbased score following algorithm with HMM-based one. Our method reports less mean error values for 16 out of 20 songs than the existing score following algorithm Antescofo. 
The absolute mean errors are reduced by $69 \%$ compared with Antescofo on average over the all songs.

There can be observed striking errors in songs ID 6-14. Main reasons are twofold. (1) In songs ID 6-10, a guitar or multiple instruments are used. Among their polyphonic sounds, some musical notes sound so vague or persist so long that the audio spectrogram becomes different from the GMM-based spectrogram generated by (27). Figure 6 illustrates an example that the previously performed musical notes affect the audio-to-score matching process. Although the red peaks, the score GMM peaks, matches some peaks of the audio spectrum in the blue line, the remainder energy from previous notes reduces the KL-divergence between these two spectra. (2) On top of the first reason, temporal fluctuation is observed in songs ID 11-14. These two factors lead both score following algorithms to fail to track a musical audio signal.

In most cases, our method outperforms the existing hybrid HMM-based score following Antescofo. These results imply that the estimation should be carried out on the audio buffer that has a certain length rather than just a frame when the music includes multiple instruments and complex polyphonic sounds. A HMM can fail to match the score with the audio because it observes just one frame when it updates the estimate of the score position. Our approach is to make use of the audio buffer to robustly match the score with the audio signal or estimate the tempo of the music.

There is a tradeoff about the length of the audio buffer $L$ or filtering interval $\Delta T$ : Longer buffer length $L$ makes the estimation of score position robust against such mismatches between the audio and score as Figure 6. Longer filtering interval $\Delta T$ allows more computational time for each filtering step. However, since our method assumes the tempo is stable in buffered $L$, larger $L$ could affect the matching between the audio and score due to a varying tempo. Also, larger $\Delta T$ causes a slow response to the tempo change. One way to reduce the trade-off is to allow for the tempo transition in the state transition model (16) and the alignment of the audio buffer with the score for the weight calculation (19).

4.2.2. The Effect of Two-Level Switching. Table 3 shows the rate of the duration where the absolute prediction error $\left|e^{\text {pred }}(t)\right|$ is limited. The leftmost column represents the ID of the song. The next three columns indicate the duration rate where $\left|e^{\text {pred }}(t)\right|<0.5$ (sec). The middle three columns indicate the duration rate where $\left|e^{\text {pred }}(t)\right|<1$ (sec). The most right-hand three columns show the duration rate where $\left|e^{\text {pred }}(t)\right|<1$ (sec) calculated from the outputs of Antescofo. For example, when the length of the song is $100(\mathrm{sec})$ and the prediction error is less than $1(\mathrm{sec})$ for $50(\mathrm{sec})$ in total, the duration rate where $\left|e^{\text {pred }}(t)\right|<1$ is 0.5 . Note that the values in $\left|e^{\text {pred }}(t)\right|<1$ are always more than the values in $\left|e^{\text {pred }}(t)\right|<0.5$ in the same configurations. The column " 30 " means that the rate is calculated from the first $30(\mathrm{sec})$ of the song. The column " 60 " uses the first 60 (sec), and "all" uses the full length of the song. For example, when the prediction error is less than $1(\mathrm{sec})$ for 27 seconds in the first

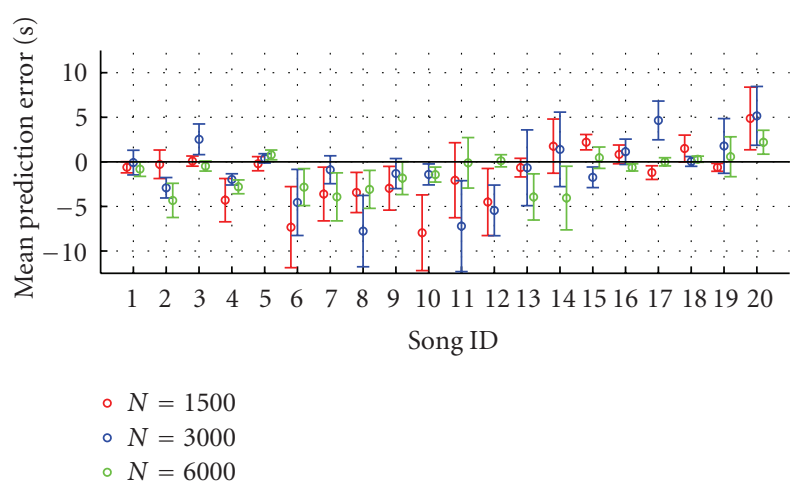

Figure 7: Number of particles $N$ versus prediction errors.

30 seconds, the rate in $\left|e^{\text {pred }}(t)\right|<1$, “ $\sim 30$ " column becomes 0.9. Bold values in the middle three columns indicate that our method outperforms Antescofo on the given condition.

Table 3 also shows that the duration of low error decreases as the incremental estimation proceeds. This is because the error in the incremental alignment is cumulative. The end of the part of a song is apt to be false aligned.

Table 4 shows the rate of the duration where the absolute prediction error $\left|e^{\text {pred }}(t)\right|<1$ (sec) on the melody level, or where the tempo estimation error is less than 5 (bpm). That is, $\left|\mathrm{BPM}-60 / \hat{b}_{n}\right|<5$, where BPM is the true tempo of the song in question. In each cell of three columns at the center, the ratio of duration that holds $\left|e^{\text {pred }}(t)\right|<1$ on the melody level is written in the left and the ratio of duration that holds $\left|\mathrm{BPM}-60 / \hat{b}_{n}\right|<5$ on the rhythm level is written in the right. The rightmost column shows the duration rate of the melody level throughout the music, which corresponds to the "all" column. "N/A" on the rhythm level indicates that there is no rhythm level output. Bold values indicate the rate is over that of both levels in Table 3 on the same condition. On the other hand, underlined values are under the rate of both levels.

The switching mechanism has a tendency to filter out erroneous estimation of the score position especially when the alignment error is cumulative because more bold values are seen in the "all" column. However, there still remains some low rates such as song IDs $4,8-10,16$. In these cases our score follower loses the part and accumulates the error dramatically, and therefore, the switching strategy becomes less helpful.

4.2.3. Prediction Error versus the Number of Particles. Figure 7 shows the mean prediction errors for various numbers of particles $N$ on both levels. For each song, the mean and standard deviation of signed prediction errors $e^{\text {pred }}(t)$ are plotted with three configurations of $N$. In this experiment, $N$ is set to $N=1500,3000,6000$.

This result implies our method is hardly improved by simply using a larger number of particles. If the state transition model and observation model match the audio signal, the error should converge to 0 with the increased number of particles. This is probably because the erroneous estimation is caused by the mismatch between the audio and 
TABLE 3: Score following error ratio w/o level switching.

\begin{tabular}{|c|c|c|c|c|c|c|c|c|c|}
\hline \multirow[b]{3}{*}{ song ID } & \multicolumn{6}{|c|}{ The range of the evaluation (sec) } & \multicolumn{3}{|c|}{ Antescofo results } \\
\hline & $\sim 30$ & $\sim 60$ & all & $\sim 30$ & $\sim 60$ & all & $\sim 30$ & $\sim 60$ & all \\
\hline & \multicolumn{3}{|c|}{$\left|e^{\text {pred }}(t)\right|<0.5(\mathrm{sec})$} & \multicolumn{3}{|c|}{$\left|e^{\text {pred }}(t)\right|<1(\mathrm{sec})$} & \multicolumn{3}{|c|}{$\left|e^{\operatorname{pred}}(t)\right|<1(\sec )$} \\
\hline 1 & 0.87 & 0.52 & 0.33 & 1.00 & 0.97 & 0.70 & 0.06 & 0.04 & 0.02 \\
\hline 2 & 0.40 & 0.33 & 0.16 & 0.80 & 0.82 & 0.39 & 0.63 & 0.73 & 0.38 \\
\hline 3 & 0.83 & 0.65 & 0.57 & 1.00 & 1.00 & 0.92 & 0.04 & 0.02 & 0.01 \\
\hline 4 & 0.10 & 0.05 & 0.02 & 0.20 & 0.10 & 0.04 & 0.18 & 0.08 & 0.03 \\
\hline 5 & 1.00 & 0.95 & 0.62 & 1.00 & 1.00 & 0.79 & 0.41 & 0.22 & 0.09 \\
\hline 6 & 0.40 & 0.20 & 0.07 & 0.63 & 0.32 & 0.12 & 0.69 & 0.47 & 0.16 \\
\hline 7 & 0.57 & 0.38 & 0.16 & 0.90 & 0.63 & 0.26 & 0.24 & 0.12 & 0.04 \\
\hline 8 & 0.43 & 0.22 & 0.05 & 1.00 & 0.52 & 0.13 & 0.25 & 0.17 & 0.05 \\
\hline 9 & 0.40 & 0.22 & 0.09 & 0.70 & 0.43 & 0.19 & 0.53 & 0.24 & 0.06 \\
\hline 10 & 0.57 & 0.28 & 0.07 & 0.87 & 0.45 & 0.11 & 0.19 & 0.11 & 0.02 \\
\hline 11 & 0.07 & 0.15 & 0.18 & 0.43 & 0.72 & 0.43 & 0.75 & 0.68 & 0.42 \\
\hline 12 & 0.33 & 0.47 & 0.11 & 0.73 & 0.85 & 0.19 & 0.70 & 0.23 & 0.10 \\
\hline 13 & 0.57 & 0.42 & 0.32 & 1.00 & 0.75 & 0.64 & 0.11 & 0.04 & 0.01 \\
\hline 14 & 0.23 & 0.32 & 0.22 & 0.47 & 0.60 & 0.40 & 0.61 & 0.37 & 0.10 \\
\hline 15 & 0.07 & 0.03 & 0.02 & 0.37 & 0.18 & 0.08 & 0.05 & 0.02 & 0.01 \\
\hline 16 & 0.80 & 0.53 & 0.30 & 1.00 & 0.88 & 0.56 & 0.57 & 0.35 & 0.16 \\
\hline 17 & 0.30 & 0.15 & 0.18 & 0.47 & 0.25 & 0.28 & 0.36 & 0.17 & 0.10 \\
\hline 18 & 0.93 & 0.88 & 0.31 & 1.00 & 1.00 & 0.42 & 0.16 & 0.09 & 0.03 \\
\hline 19 & 0.27 & 0.52 & 0.38 & 1.00 & 1.00 & 0.86 & 0.55 & 0.30 & 0.10 \\
\hline 20 & 0.73 & 0.55 & 0.18 & 1.00 & 0.78 & 0.25 & 0.03 & 0.01 & 0.02 \\
\hline
\end{tabular}

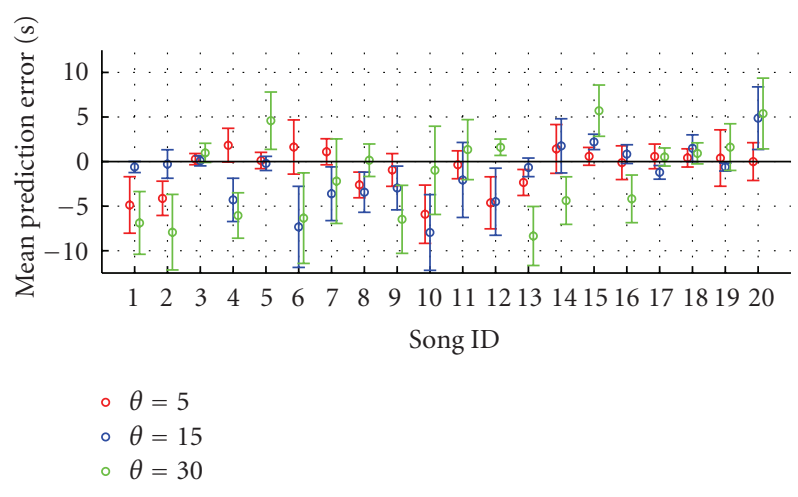

Figure 8: Window width $\theta$ versus prediction errors.

score as shown in Figure 6. Considering that the estimation results have not been saturated after increasing the particles, the performance can converge by adding more particles such as thousands or even millions of particles.

4.2.4. Prediction Error versus the Width of the Tempo Window. Figure 8 shows the mean and standard deviation of signed prediction errors for various widths of tempo window $\theta$. In this experiment, $\theta$ is set to 5,15 , and 30 (bpm).
Intuitively, the narrower the width is, the closer to zero the error value should be because the chance of choosing a wrong tempo will be reduced.

However, the prediction errors are sometimes unstable, especially for those IDs under 10 which has no drums, because the width is too narrow to account for the temporal fluctuations in the actual performance. The musical performance tends to temporally fluctuate without drums or percussions. On the other hand, the prediction errors for IDs 11-20 are less when the width is narrower. This is because the tempo in the audio signal is stable thanks to the drummer. In particular, stable and periodic drum onsets in IDs 15-20 make the peaks in the normalized cross correlation in (10) sufficiently striking to choose a correct beat interval value from the proposal distribution in (5). This result confirms that our method reports less error with stable drum sounds even though drum sounds tend to cover the harmonic structure of pitched sounds.

4.3. Computational Cost in Our Algorithm. The procedure that requires the computational resource most in our method is the observation process. In particular, the harmonic structure matching consumes the processor time as described in (25) and (26). The complexity of this procedure conforms to $O\left(N L f_{\max }\right)$, where $N$ is the number of particles, $L$ is the length of the spectrogram, and $f_{\max }$ is the range of the frequency considered in the matching. 
TABLE 4: Score following error ratio w/ level switching. Left: melody level accuracy, $\left|e^{\text {pred }}(t)\right|<1$ (sec). Right: rhythm level accuracy, $\left|B P M-60 / \hat{b}_{n}\right|<5$ (bpm).

\begin{tabular}{cccccccc}
\hline \multicolumn{6}{c}{ The range of the evaluation (sec) } & $\begin{array}{c}\text { Melody level } \\
\text { song ID }\end{array}$ & \multicolumn{2}{c}{$\sim$ No } & \multicolumn{2}{c}{$\sim 60$} & all & ratio \\
\hline 1 & 1.00 & N/A & 0.97 & N/A & 0.70 & N/A & 1.00 \\
2 & 0.80 & N/A & 0.82 & N/A & 0.39 & 1.00 & 0.99 \\
3 & 1.00 & N/A & 1.00 & N/A & $\mathbf{0 . 9 3}$ & 1.00 & 0.99 \\
4 & 0.20 & N/A & 0.10 & N/A & 0.04 & N/A & 1.00 \\
5 & 1.00 & N/A & 1.00 & N/A & $\mathbf{0 . 9 3}$ & 0.70 & 0.71 \\
6 & $\mathbf{0 . 7 2}$ & 1.00 & $\mathbf{0 . 7 2}$ & 1.00 & $\mathbf{0 . 6 8}$ & 0.95 & 0.19 \\
7 & $\mathbf{0 . 9 6}$ & 0.50 & $\mathbf{0 . 6 8}$ & 0.70 & $\mathbf{0 . 3 5}$ & 0.40 & 0.55 \\
8 & 1.00 & 0.44 & $\mathbf{1 . 0 0}$ & 0.24 & $\underline{0.04}$ & 0.14 & 0.56 \\
9 & $\underline{0.50}$ & 0.69 & $\mathbf{0 . 5 0}$ & 0.89 & $\underline{0.12}$ & 0.92 & 0.60 \\
10 & $\mathbf{1 . 0 0}$ & 1.00 & $\underline{0.43}$ & 1.00 & $\mathbf{0 . 1 5}$ & 0.71 & 0.62 \\
11 & 0.43 & N/A & 0.72 & N/A & $\mathbf{0 . 5 9}$ & 0.25 & 0.51 \\
12 & 0.73 & N/A & 0.85 & N/A & $\mathbf{0 . 2 5}$ & 0.71 & 0.76 \\
13 & 1.00 & 1.00 & $\mathbf{0 . 7 8}$ & 1.00 & $\mathbf{0 . 7 2}$ & 1.00 & 0.55 \\
14 & $\underline{0.45}$ & 0.38 & $\underline{0.48}$ & 0.70 & $\underline{0.20}$ & 0.84 & 0.44 \\
15 & $\mathbf{1 . 0 0}$ & 0.22 & $\mathbf{0 . 2 7}$ & 0.20 & $\underline{0.05}$ & 0.25 & 0.43 \\
16 & 1.00 & 0.42 & $\underline{0.77}$ & 0.29 & $\underline{0.48}$ & 0.31 & 0.81 \\
17 & 0.60 & N/A & 0.33 & N/A & 0.34 & N/A & 1.00 \\
18 & 1.00 & N/A & 1.00 & N/A & 0.42 & N/A & 1.00 \\
19 & 1.00 & N/A & 1.00 & 1.00 & $\mathbf{1 . 0 0}$ & 1.00 & 0.53 \\
20 & 1.00 & 0.71 & $\mathbf{0 . 8 4}$ & 0.29 & $\mathbf{0 . 3 6}$ & 0.38 & 0.54 \\
\hline
\end{tabular}

For real-time processing, the whole particle filtering process must be done in $\Delta T$ (sec) because the filtering process takes place every $\Delta T(\mathrm{sec})$. The observation process, namely, the weight calculation for each particle, can be parallelized because the weight of each particle is independently evaluated. Therefore, we can reduce the complexity to $O\left(N L f_{\max } / Q_{\mathrm{MT}}\right)$, where $\mathrm{Q}_{\mathrm{MT}}$ denotes the number of threads for the observation process.

Figure 9 shows the real-time factors in various configurations of the particle number $N$ and the number of threads $Q_{\text {MT }}$. These curves confirm that the computational time grows in proportion to $N$ and reduces in inverse proportion to $Q_{\mathrm{MT}}$.

\section{Discussion and Future Work}

Experimental results show that the score following performance varies with the music played. Needless to say, a music robot hears a mixture of musical audio signals and its own singing voice or instrumental performance. Some musical robots $[7,11,27]$ use self-generating sound cancellation [28] from a mixture of sounds. Our score following should be tested with such cancellation because the performance of score following may deteriorate if such cancellation is used.

The design of the two-level synchronization is intended to improve existing methods reported in the literature. There is a trade-off between a tempo tracking and a score following: the tempo tracking result is accurate when drum

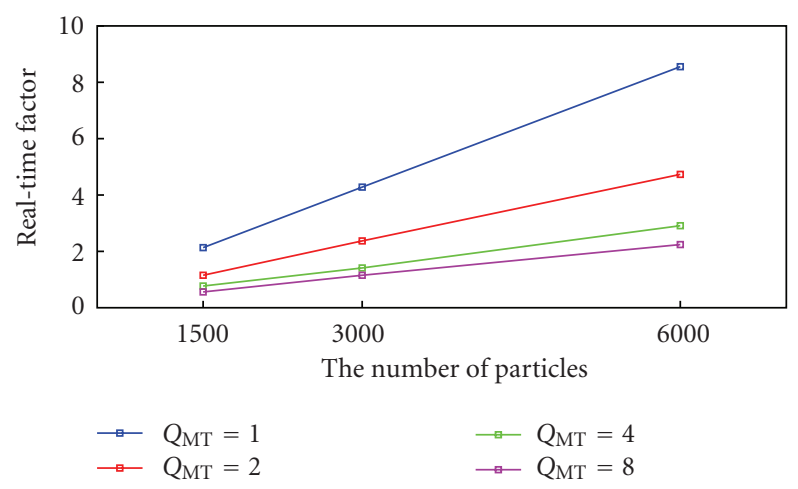

FIgURE 9: Real-time factor curve.

or percussive sounds are included in the audio signal, while the score following result is sometimes deteriorated by these percussive sounds because those sounds conceal the harmonic structure of pitched instruments.

To make a musical expression on the rhythm level, the robot might require not only the beat interval but also the beat time. To estimate both the beat time and beat interval for the rhythm level interaction, a state space model for the beat tracking will be an effective solution [29]. An extension of our model to estimate the beat interval, score position, and beat time can be enumerated as one of the future works. The switching whether the beat time or the score position along with the beat interval can be determined by the estimation confidence.

5.1. Future Works. The error in the estimation of the score position accumulates as the audio signal is incrementally input. We present the two-level switching mechanism to cope with this situation. Another solution is error recovery by landmark search. When we listen to the music and lose the part being played, we often pay attention to find a landmark in the song, for example, the beginning of the chorus part. After finding the landmark, we can start singing or playing our instrument again. The framework of a particle filter enables us to realize the idea of this landmark search-based error recovery by modifying the proposal distribution. When a landmark is likely to be found in the input audio signal, the score follower can jump to the corresponding score position by adding some particle at the point. The issues in this landmark search are landmark extraction from the musical score and the incremental detection of the landmarks from the audio signal.

There remains a limitation in our framework: Our current framework assumes that the input audio signal is performed in the same way as written in the score. Some musical scores, for example, jazz scores, provide only abstract notations such as chord progressions. Tracking the audio with these abstract notations is one of further challenges.

There are other aspects of the advantages in the use of the particle filter for a score following. Our score following using the particle filter should also be able to improve an instrument-playing robot. In fact, a theremin player robot moves its arms to determine the pitch and the volume 
of theremin. Therefore, the prediction mechanism enables the robot to play the instrument in synchronization with the human performance. In addition, a multimodal ensemble system using a camera [30] can be naturally aggregated with our particle-filter-based score following system. Several music robots use a camera to acquire visual cues from human musicians $[8,31]$. This is because the flexible framework of the particle filter facilitates aggregation of multimodal information sources [32].

We are currently developing ensemble robots with a human flutist. The human flutist leads the ensemble, and a singer and thereminist robot follows [31]. The two-level synchronization approach benefits this ensemble as follows: when the score position is uncertain, the robot starts scatting the beats, or faces downward and sings in a low voice; when the robot is aware of the part of the song, it faces up and presents a loud and confident voice. This posture-based voice control is attained through the voice manipulation system [33].

Another application of score following is automatic page turning of the musical score $[15,34]$. In particular, automatic page turning systems running on portable tablet computers like the iPad, developed by Apple Computer Inc., would be convenient for daily practice of musical instruments where both hands are required to play, such as piano or guitar. Further reduction of computational cost is important to run the score following algorithm on portable tablet computers that have limited memory and a less powerful processor.

\section{Conclusion}

Our goal is to develop a coplayer music robot that presents musical expressions in accordance with a human's musical performance. The synchronization function is essential for a coplayer robot. This paper presented a score following system based on a particle filter to attain the two-level synchronization for interactive coplayer music robots. Our method make use of the onset information and the prior knowledge about the tempo provided by the musical score by modeling proposal distributions for the particle filter. Furthermore, to cope with an erroneous estimation, twolevel synchronization is performed at the rhythm level and the melody level. The reliability to switch between the two levels of score following is calculated from the density of particles and is used to switch between levels.

Experiments were carried out using 20 jazz songs performed by human musicians. The experimental results demonstrated that our method outperforms the existing score following system called Antescofo in 16 songs out of 20. The error in the prediction of the score position is reduced by $69 \%$ on average compared with Antescofo. The results also revealed that the switching mechanism alleviates the error in the estimation of the score position, although the mechanism becomes less effective when the error is accumulated and the follower loses the part being played.

One possible solution to the cumulative error in the incremental alignment of the audio with the score is a landmark search. Our particle filter framework would naturally take this into account as a proposal distribution of landmark detection. The future work will also include development of interactive ensemble robots. In particular, multimodal synchronization function using both the audio and visual cues would enrich the human-robot musical ensemble dramatically.

\section{Acknowledgments}

This research was supported in part by Kyoto University Global COE, in part by JSPS Grant-in-Aid for Scientific Research (S) 19100003, and in part by a Grant-in-Aid for Scientific Research on Innovative Areas (no. 22118502) from the MEXT, Japan. The authors would like to thank LouisKenzo Cahier and Angelica Lim for beneficial comments on earlier drafts, and the members of Okuno and Ogata Laboratory for their discussion and valuable suggestions.

\section{References}

[1] Y. Kusuda, "Toyota's violin-playing robot," Industrial Robot, vol. 35, no. 6, pp. 504-506, 2008.

[2] A. Alford, S. Northrup, K. Kawamura, K.-W. Chan, and J. Barile, "A music playing robot," in Proceedings of the International Conference on Field and Service Robotics (FSR '99), pp. 29-31, 1999.

[3] K. Shibuya, S. Matsuda, and A. Takahara, "Toward developing a violin playing robot-bowing by anthropomorphic robot arm and sound analysis," in Proceedings of the 16th IEEE International Conference on Robot and Human Interactive Communication (RO-MAN '07), pp. 763-768, August 2007.

[4] S. Kotosaka and S. Shaal, "Synchronized robot drumming by neural oscillator," Journal of Robotics Society of Japan, vol. 19, no. 1, pp. 116-123, 2001.

[5] H. Kozima and M. P. Michalowski, "Rhythmic synchrony for attractive human-robot interaction," in Proceedings of the Entertainment Computing, 2007.

[6] K. Yoshii, K. Nakadai, T. Torii et al., "A biped robot that keeps steps in time with musical beats while listening to music with its own ears," in Proceedings of the IEEE/RSJ International Conference on Intelligent Robots and Systems, pp. 1743-1750, 2007.

[7] K. Murata, K. Nakadai, K. Yoshii et al., "A robot uses its own microphone to synchronize its steps to musical beats while scatting and singing," in Proceedings of the IEEE/RSJ International Conference on Intelligent Robots and Systems, pp. 2459-2464, 2008.

[8] Y. Pan, M. G. Kim, and K. Suzuki, "A robot musician interacting with a human partner through initiative exchange," in Proceedings of the Conference on New Interfaces for Musical Expression (NIME '10), pp. 166-169, 2010.

[9] G. Weinberg and S. Driscoll, "Toward robotic musicianship," Computer Music Journal, vol. 30, no. 4, pp. 28-45, 2006.

[10] R. B. Dannenberg and C. Raphael, "Music score alignment and computer accompaniment," Communications of the ACM, vol. 49 , no. 8 , pp. $39-43,2006$.

[11] T. Otsuka, K. Nakadai, T. Takahashi, K. Komatani, T. Ogata, and H. G. Okuno, "Incremental polyphonic audio to score alignment using beat tracking for singer robots," in Proceedings 
of the IEEE/RSJ International Conference on Intelligent Robotsand Systems, pp. 2289-2296, 2009.

[12] M. S. Arulampalam, S. Maskell, N. Gordon, and T. Clapp, "A tutorial on particle filters for online nonlinear/non-Gaussian Bayesian tracking," IEEE Transactions on Signal Processing, vol. 50, no. 2, pp. 174-188, 2002.

[13] H. Kenmochi and H. Ohshita, "Vocaloid-commercial singing synthesizer based on sample concatenation," in Proceedings of the Interspeech Conference, pp. 4010-4011, 2007.

[14] S. Dixon, "An on-line time warping algorithm for tracking musical performances," in Proceedings of the International Joint Conference on Artificial Intelligence, pp. 1727-1728, 2005.

[15] A. Arzt, G. Widmer, and S. Dixon, "Automatic page turning for musicians via real-time machine listening," in Proceedings of the European Conference on Artificial Intelligence, pp. 241$245,2008$.

[16] N. Orio, S. Lemouton, and D. Schwarz, "Score following: state of the art and new developments," in Proceedings of the International Conference on New Interfaces for Musical Expression, pp. 36-41, 2003.

[17] A. Cont, D. Schwarz, and N. Schnell, "Training IRCAM's score follower," in Proceedings of the AAAI Fall Symposium on Style and Meaningin Art, Language and Music, 2004.

[18] A. Cont, "ANTESCOFO: anticipatory synchronization and control of interactive parameters in computer music," in Proceedings of the International Computer Music Conference, 2008.

[19] C. Raphael, "Aligning music audio with symbolic scores using a hybrid graphical model," Machine Learning, vol. 65, no. 2-3, pp. 389-409, 2006.

[20] O. Izmirli, R. Seward, and N. Zahler, "Melodic pattern anchoring for score following using score analysis," in Proceedings of the International Computer Music Conference, pp. 411-414, 2003.

[21] T. Otsuka, K. Nakadai, T. Takahashi, K. Komatani, T. Ogata, and H. G. Okuno, "Design and implementation of two-level synchronization for interactive music robot," in Proceedings of the24th AAAI Conference on Artificial Intelligence, pp. 12381244, 2010.

[22] J. P. Bello, C. Duxbury, M. Davies, and M. Sandler, "On the use of phase and energy for musical onset detection in the complex domain," IEEE Signal Processing Letters, vol. 11, no. 6, pp. 553556, 2004.

[23] J. P. Bello, L. Daudet, S. Abdallah, C. Duxbury, M. Davies, and M. B. Sandler, "A tutorial on onset detection in music signals," IEEE Transactions on Speech and Audio Processing, vol. 13, no. 5, pp. 1035-1046, 2005.

[24] M. Goto, "A chorus section detection method for musical audio signals and its application to a music listening station," IEEE Transactions on Audio, Speech and Language Processing, vol. 14, no. 5, pp. 1783-1794, 2006.

[25] A. Cont, "A coupled duration-focused architecture for realtime music-to-score alignment," IEEE Transactions on Pattern Analysis and Machine Intelligence, vol. 32, no. 6, pp. 974-987, 2010.

[26] M. Goto, H. Hashiguchi, T. Nishimura, and R. Oka, "RWC music database: music genre database and musical instrument sound database," in Proceedings of the International Conference on Music Information Retrieval, pp. 229-230, 2003.

[27] T. Otsuka, K. Nakadai, T. Takahashi, K. Komatani, T. Ogata, and H. G. Okuno, "Music-ensemble robot that is capable of playing the theremin while listening to the accompanied music," in Proceedings of the International Conference on Industrial, Engineering and Other Applications of Applied
Intelligent Systems (IEA/AIE '10), vol. 6096 of Lecture Notes in Artificial Intelligence, pp. 102-112, 2010.

[28] R. Takeda, K. Nakadai, K. Komatani, T. Ogata, and H. G. Okuno, "Barge-in-able robot audition based on ICA and missing feature theory under semi-blind situation," in Proceedings of the IEEE/RSJ International Conference on Intelligent Robotsand Systems, pp. 1718-1723, 2008.

[29] A. T. Cemgil and B. Kappen, "Monte Carlo methods for tempo tracking and rhythm quantization," Journal of Artificial Intelligence Research, vol. 18, pp. 45-81, 2003.

[30] D. Overholt, J. Thompson, L. Putnam et al., "A multimodal system for gesture recognition in interactive music performance," Computer Music Journal, vol. 33, no. 4, pp. 69-82, 2009.

[31] A. Lim, T. Mizumoto, L. Cahier et al., "Robot musical accompaniment: integrating audio and visual cues for realtime synchronization with a human flutist," in Proceedings of the IEEE/RSJ International Conference on Intelligent Robots and Systems, 2010.

[32] K. Nickel, T. Gehrig, R. Stiefelhagen, and J. McDonough, "A joint particle filter for audio-visual speaker tracking," in Proceedings of the International Conference on Multimodal Interfaces, pp. 61-68, 2005.

[33] T. Otsuka, K. Nakadai, T. Takahashi, K. Komatani, T. Ogata, and H. G. Okuno, "Voice-awareness control for a humanoid robot consistent with its body posture and movements," PALADYN Journal of Behavioral Robotics, vol. 1, no. 1, pp. 80$88,2010$.

[34] R. Dannenberg, M. Sanchez, A. Joseph, P. Capell, R. Joseph, and R. Saul, "A computer-based multi-media tutor for beginning piano students," Interface Journal of New Music Research, vol. 19, no. 2-3, pp. 155-173, 1993. 